\title{
Descentralización de la actividad científica. El papel determinante de las regiones centrales: el caso de Madrid
}

\author{
Fernanda Morillo* y Daniela De Filippo*
}

Resumen: En los últimos años se ha advertido una tendencia creciente a la descentralización regional en la producción científica de España, ya que Madrid está perdiendo gradualmente su papel dominante. Para analizar este proceso, en el presente estudio se plantean una serie de interrogantes como: ¿cuáles son las regiones que más aumentan su producción científica?, ¿qué importancia tiene la cooperación con Madrid en este crecimiento? Se estudia también si la colaboración con Madrid contribuye a mejorar el impacto y la visibilidad de las publicaciones. Para dar respuesta a estas cuestiones se analiza la producción española a través de las bases de datos internacionales del Web of Science (período 2001-2005). Los principales resultados obtenidos muestran que las regiones que más aumentaron su producción fueron la Comunidad Valenciana y Andalucía pero, proporcionalmente, el crecimiento más excepcional lo experimentó Castilla-La Mancha. Aunque la colaboración con Madrid es muy significativa, Cataluña también es un importante centro de atracción. Sin embargo, se ha detectado que la cooperación con Madrid mejora el impacto y la visibilidad especialmente en Medicina Clínica y que sigue siendo un polo importante de desarrollo científico cuyo apoyo a las regiones más pequeñas resulta fundamental.

Palabras clave: Web of Science, colaboración regional, Madrid, análisis de redes.

\section{Decentralization of scientific activity. The major role of central regions: the case of Madrid}

Abstract: In recent years there has been an increasing trend towards the decentralization of Spanish scientific production, with Madrid gradually losing its dominant role. To examine this process, the present study raises a series of questions, such as which regions are most increasing their scientific output?, and what is the importance of cooperation with Madrid in this growth? Whether collaboration with Madrid contributes towards improving the impact and visibility of publications is also examined. To answer these questions, Spanish scientific production was analysed using the Web of Science international databases (period 2001-2005).The results show that the Valencia and Andalusia regions are those which have most increased their scientific production, but proportionally the most exceptional growth was achieved in Castilla-La Mancha. Although collaboration with Madrid is very significant, Catalonia is also an important region in this respect. However, cooperation with Madrid especially improved the impact

* IEDCYT (CCHS), CSIC. Correo-e: fernanda.morillo@cchs.csic.es, daniela.defilippo@cchs.csic.es. Recibido: 15-10-08; 2. ${ }^{a}$ versión: 24-3-09. 
and visibility of publications in the area of clinical medicine, and this region continues to be an important centre of scientific development, the support of which is vital to the growth of output in the smallest regions of Spain.

Keywords: Web of Science, regional collaboration, Madrid, network analysis

\section{Introducción}

Una característica importante del quehacer científico es la tendencia creciente al trabajo en grupo. La colaboración puede ocurrir entre instituciones, disciplinas, sectores, regiones o países. Si bien se ha debatido mucho sobre los beneficios de la colaboración, su estudio resulta de plena actualidad debido al importante papel que juega en la actividad científica. Autores como Beaver (2001), han profundizado en el estudio de las razones que llevan a cooperar y acentúan la importancia de los factores económicos (los altos costes de instrumentos y equipo en ciertas áreas hacen la cooperación necesaria) y políticos (ayuda a la investigación y la colaboración científica por parte de gobiernos y organismos regionales o internacionales). La motivación para cooperar se ve influida por las metas de aquéllos que proporcionan la financiación, la necesidad de tener acceso al conocimiento o a los equipos, o las oportunidades que favorecen el trabajo conjunto (p. ej., proximidad geográfica).

La colaboración científica aumenta el número de contactos entre investigadores y puede realzar la visibilidad potencial de una publicación. En este sentido, diferentes estudios han mostrado los beneficios de la colaboración en función del impacto y la visibilidad que pueden alcanzar las publicaciones (Moed, 2000) y han detectado una posible relación entre la colaboración internacional y el aumento de la calidad de estas publicaciones (Van Raan, 1998). Sin embargo, como explica Glänzel (2001), la influencia de la colaboración internacional en el impacto de las publicaciones de un país puede variar considerablemente entre diferentes países y campos temáticos. Un claro ejemplo lo constituyen los datos aportados por un estudio reciente (Gómez y otros, 2009) en el que se analiza la cooperación de los países Latinoamericanos con los europeos. En él se advierte que la influencia de la UE produce un incremento de la visibilidad (en cuanto al número de citas recibidas) entre 2 y 3 veces mayor que la producción doméstica, en todas las áreas. Se ha detectado que las áreas más beneficiadas son especialmente Física y Medicina Clínica. Este diferente impacto de la colaboración en cada área temática podría explicar, tal como menciona Wagner (2005), por qué la estructura de las redes de colaboración establecidas y sus tipologías están tan ligadas a los campos implicados.

A pesar del gran impacto que parece tener la colaboración, autores como Godin e Ippersiel (1996) argumentan que todavía sabemos muy poco sobre formas de colaboración científica como la regional. Esta carencia de estudios bibliométricos regionales (aparte de los de la Unión Europea) es asombrosa a la luz 
de la importancia de esta dimensión en políticas de ciencia y tecnología. Los autores detectaron que la colaboración regional es muy limitada debido, quizás, a la creciente internacionalización de la ciencia y al "prestigio" científico que parece aportar la colaboración con instituciones de otros países. La mayor razón para cooperar puede darse, además de por la proximidad geográfica o lingüística, por el pequeño tamaño del grupo o institución productora. Esto se evidencia en su estudio sobre la producción científica en Canadá. En él los autores demuestran que las regiones periféricas tienen el mayor aumento de documentos a lo largo de los años y el centro (en su caso, Montreal) es en gran parte autónomo, presentando menos colaboración con otras regiones que la que proporcionalmente tienen las demás con él.

Esta noción de centro y periferia, en términos de producción científica, es la que lleva —según se aprecia en el estudio de Schubert y Glänzel (2006) — a presentar a países como EE.UU. como "centro" científico. Esto se debe al papel destacado que juega en la productividad internacional y a la preferencia universal que los otros países muestran hacia él al elegirlo como principal colaborador.

En España, algo similar parece ocurrir con Madrid, que se presenta como un importante centro de atracción. Sin embargo, en los últimos años se ha detectado que otras regiones están aumentando su participación en la producción española, mientras que la de Madrid está disminuyendo (Gómez y otros, 2007) de una manera similar a lo que sucede con EE.UU. respecto de la producción mundial (Leydesdorff y Wagner, 2006). Esto —en el caso español- podría deberse a la creación de nuevos centros en otras regiones y a una tendencia a una distribución más homogénea de la producción científica del país.

En este trabajo se quiere conocer la importancia de regiones centrales como Madrid para el desarrollo de la actividad científica del resto de las regiones. Para ello se intentará dar respuesta a algunos de los siguientes interrogantes:

- ¿Qué regiones son las que más crecen?

- ¿Es Madrid responsable del incremento de las regiones más destacadas?

Para estudiar si Madrid tiene efecto también en el impacto y la visibilidad de otras regiones, se plantean otras dudas:

- ¿La colaboración con Madrid aumenta el impacto y la visibilidad de estas otras regiones por áreas y disciplinas?

- ¿El mayor impacto se relaciona, además, con una mayor colaboración internacional?

Finalmente, se estudia también en qué medida el análisis de redes sociales se presenta como una herramienta óptima para visualizar las complejas relaciones entre comunidades y reflejar los efectos que la colaboración con Madrid tiene por áreas y disciplinas. 


\section{Metodología}

Los resultados de la investigación española se analizan a través de las bases de datos internacionales Web of Science (WoS) de Thomson-Reuter (SCI, SSCI y A\&HCI) en el período 2001-2005. Se seleccionan estas bases de datos ya que son una de las principales vías de difusión utilizadas por los investigadores españoles. Asimismo, dado que estas fuentes aportan información sobre los centros de investigación de todos los autores firmantes, es posible analizar la influencia de la colaboración. Todo el estudio que se presenta a continuación se centra en el quinquenio mencionado pero, para disponer de una serie temporal más amplia al analizar la evolución de las regiones españolas (Tabla I en los resultados), se ha contado con datos de los años 1996-2000 obtenidos de las bases de datos en formato CD-ROM. Aunque se trata de una fuente distinta, las pruebas estadísticas muestran correlación con los datos WoS y, por ello, resulta apta para presentar la evolución de la producción internacional en la última década.

Si bien existen diferentes métodos para la asignación de documentos, se ha optado por el recuento total, ya que permite conocer la participación real de un centro o región, mientras que el recuento fraccionado hace referencia a la aportación y penaliza la colaboración. Asimismo, mediante pruebas estadísticas se detectó correlación entre ambos métodos de recuento.

Una vez tratados los datos, se obtuvieron los siguientes indicadores:

- Indicadores de actividad: evolución anual del número de documentos de cada región y número total de publicaciones por áreas y disciplinas. Para esto, se ha seguido la clasificación disciplinar que hace WoS de las revistas y se han agrupado en grandes áreas a partir de criterios similares a los del Current Contents. En este caso se ha excluido el área de Humanidades (por carecer sus revistas de factor de impacto) y las categorías multidisciplinares. Se considera también la colaboración que se produce entre las distintas regiones.

- Indicadores de citación e impacto: se ha obtenido el número de citas por documento y se han calculado también las citas relativas $(C R)$ comparando los valores de cada región con los del promedio de España. En este caso la ventana de citación empleada va desde la fecha de publicación del documento hasta el año 2006. Utilizando el factor de impacto del 2005 se ha calculado el factor de impacto medio de cada área y/o región y el factor de impacto relativo $(F I R)$ a la media del país.

Para el cálculo de las correlaciones estadísticas se ha utilizado el software 15.0 de SPSS. Para mostrar los resultados se emplearon los programas de Pajek y UCINET (Borgatti y otros, 2002). El primero se utiliza para visualizar las redes, que representan las relaciones entre nodos y son muy útiles para describir la estructura subyacente entre los diferentes actores (Batagelj y Mrvar, 2002). UCINET 
permite calcular una serie de indicadores como la densidad, que es un indicador del nivel general de conexión del gráfico. También se pueden obtener medidas de centralidad como: grado de centralidad (degree centrality), cercanía (closeness) e intermediación (betweenness). El grado de centralidad de un nodo se define como el número de enlaces que tiene. La cercanía de un nodo es igual a la distancia total (en el gráfico) de este nodo respecto al resto de nodos. Finalmente, la intermediación es el número de trayectorias más cortas que pasan por un nodo dado. Esta medida calibra hasta qué punto un nodo facilita el flujo en la red. Además, cada medida de centralidad individual se puede utilizar para derivar una medida de centralidad para la red entera (Otte y Rousseau, 2002). Estos indicadores han sido utilizados para calcular las posiciones e importancia de los diferentes nodos en la red, ya sean instituciones concretas o regiones. En todos los casos se presentan los gráficos "dirigidos", es decir, en los que el peso de la relación entre los nodos es asimétrica y se ha considerado la intensidad de la relación en función del tamaño de cada nodo.

\section{Resultados}

En este apartado se intenta dar respuesta a los interrogantes planteados anteriormente.

\section{1. ¿Qué regiones son las que más crecen?}

La Tabla I presenta la evolución de la participación regional en la producción española entre 1996 y 2005 (ver metodología). La última columna muestra el ratio entre la participación regional del último y del primer año del período. Así, valores superiores a la unidad implican un incremento de la participación regional en la producción total española. Se aprecia que Madrid pierde más del 2\%, mientras que la Comunidad Valenciana y Andalucía ganan 3 y 2 puntos porcentuales respectivamente. A pesar de esta pérdida, la proporción de la misma para Madrid no es la peor: se ve sobrepasada por el descenso de las Islas Canarias y Aragón. Por otro lado, el incremento proporcional más notorio es para Castilla-La Mancha $(1,95)$ que pasa de representar el 1,04\% de la producción Española en 1996 al 2,03\% en 2005.

\section{2. ¿Es Madrid responsable del incremento de las regiones más destacadas?}

Para analizar si el incremento de la participación de algunas regiones (Tabla I) se explica en parte por las prácticas de colaboración con Madrid, se estudia la evolución de dicha colaboración en cada región. Los datos muestran que este 


\section{TABLA I}

Evolución de la participación regional en la producción española (1996-2005)

\begin{tabular}{l|r|r|r|r|r|r|r|r|r|r|r}
\hline $\begin{array}{l}\text { Comunidad } \\
\text { Autónoma }\end{array}$ & $\mathbf{1 9 9 6}$ & $\mathbf{1 9 9 7}$ & $\mathbf{1 9 9 8}$ & $\mathbf{1 9 9 9}$ & $\mathbf{2 0 0 0}$ & $\mathbf{2 0 0 1}$ & $\mathbf{2 0 0 2}$ & $\mathbf{2 0 0 3}$ & $\mathbf{2 0 0 4}$ & $\mathbf{2 0 0 5}$ & $\begin{array}{c}\text { Ratio } \\
\mathbf{2 0 0 5} / \\
\mathbf{1 9 9 6}\end{array}$ \\
\hline Andalucía & 13,09 & 12,95 & 13,45 & 13,96 & 13,52 & 14,17 & 14,56 & 14,79 & 14,48 & 14,99 & 1,15 \\
\hline Aragón & 4,09 & 3,81 & 3,67 & 3,51 & 3,33 & 3,60 & 3,39 & 3,34 & 3,38 & 3,61 & 0,88 \\
\hline Asturias & 2,88 & 2,75 & 2,84 & 2,99 & 3,03 & 2,92 & 3,04 & 3,03 & 2,96 & 2,80 & 0,97 \\
\hline Castilla y León & 4,93 & 5,05 & 4,36 & 4,65 & 4,56 & 4,71 & 4,84 & 4,60 & 4,80 & 4,77 & 0,97 \\
\hline C.-La Mancha & 1,04 & 1,16 & 1,00 & 1,12 & 1,18 & 1,41 & 1,58 & 1,83 & 1,87 & 2,03 & 1,95 \\
\hline C. Valenciana & 9,19 & 9,09 & 9,88 & 10,06 & 10,44 & 10,89 & 10,57 & 11,36 & 11,53 & 11,94 & 1,30 \\
\hline Cantabria & 1,51 & 1,69 & 1,58 & 1,71 & 1,69 & 1,68 & 1,53 & 1,59 & 1,60 & 1,69 & 1,12 \\
\hline Cataluña & 23,73 & 24,58 & 24,02 & 24,93 & 24,29 & 23,27 & 23,75 & 24,25 & 25,60 & 25,42 & 1,07 \\
\hline Extremadura & 1,36 & 1,21 & 1,10 & 1,14 & 1,07 & 1,36 & 1,46 & 1,28 & 1,43 & 1,41 & 1,04 \\
\hline Galicia & 5,15 & 5,52 & 6,07 & 6,01 & 6,21 & 6,31 & 6,72 & 7,21 & 6,65 & 6,63 & 1,29 \\
\hline Islas Baleares & 1,09 & 1,14 & 1,17 & 1,20 & 1,08 & 1,23 & 1,37 & 1,34 & 1,49 & 1,45 & 1,33 \\
\hline Islas Canarias & 3,37 & 3,28 & 3,01 & 2,99 & 2,89 & 3,31 & 3,09 & 3,33 & 3,14 & 3,05 & 0,91 \\
\hline La Rioja & 0,23 & 0,27 & 0,23 & 0,28 & 0,35 & 0,34 & 0,33 & 0,30 & 0,31 & 0,35 & 1,52 \\
\hline C. de Madrid & 30,49 & 30,68 & 30,39 & 29,11 & 30,11 & 29,12 & 29,02 & 28,03 & 27,93 & 28,01 & 0,92 \\
\hline Murcia & 2,48 & 2,58 & 2,39 & 2,57 & 2,49 & 2,59 & 2,66 & 2,69 & 2,58 & 2,77 & 1,12 \\
\hline Navarra & 1,33 & 1,62 & 1,81 & 1,92 & 2,37 & 2,29 & 2,26 & 2,27 & 2,48 & 2,48 & 1,86 \\
\hline País Vasco & 4,12 & 4,10 & 4,33 & 3,90 & 4,03 & 4,12 & 3,81 & 4,14 & 3,88 & 3,90 & 0,95 \\
\hline
\end{tabular}

incremento de la participación de regiones como Castilla-La Mancha se debe lógicamente a un crecimiento más rápido frente a Madrid, aunque no parece estar relacionado con una mayor colaboración, lo que quiere decir que hay otros factores interactuando. Sin embargo, el crecimiento de la colaboración en general sí parece estar relacionado con el incremento de la colaboración con Madrid, como demuestran las pruebas estadísticas no paramétricas.

Para estudiar la atracción que ejerce Madrid sobre otras regiones, se realiza un análisis de la red interregional española y se calculan algunas medidas de centralidad (solamente para los datos WoS y el período 2001-2005, de ahora en adelante). En primer lugar, se obtiene la colaboración entre las diversas regiones (una red no dirigida) y se calcula el grado de centralidad de la red obtenida.

En segundo lugar, esta red no dirigida se transforma en dirigida considerando el peso de la colaboración para cada región (el porcentaje que representan esos 
documentos en colaboración con respecto al total de los documentos de cada región). Posteriormente se calcula el grado de centralidad (externo e interno) y se compara con el obtenido previamente. El grado de centralidad interno (InDegree) representa la recepción de la colaboración, existiendo correlación entre este grado y los valores de centralidad de la red no dirigida. En la Tabla II, Madrid muestra elevados valores porcentuales de InDegree incrementando la distancia con los valores de Cataluña, la otra región relevante en la red. Este papel central de Madrid se representa gráficamente en la Figura 1. En el lado contrario, La Rioja tiene los valores más bajos de grado de centralidad no dirigida (Degree) y, de la misma manera, los valores más altos de grado de centralidad dirigida externa (OutDegree).

Esto refleja que Madrid es más importante para las pequeñas regiones, aunque no hay correlación entre el tamaño de la región y el porcentaje de colaboración con Madrid. Entre las regiones a las que más atrae figura principalmente Casti-

TABLA II

Red No-Dirigida versus Dirigida (WoS 2001-2005). Medidas de grado de centralidad de Freeman (Degree), externas (OutDegree) e internas (InDegree)

\begin{tabular}{|c|c|c|c|c|c|c|}
\hline \multirow{2}{*}{$\begin{array}{l}\text { Comunidad } \\
\text { Autónoma }\end{array}$} & \multicolumn{2}{|c|}{ No-Dirigida } & \multicolumn{4}{|c|}{ Dirigida } \\
\hline & Degree & $\%$ & OutDegree & InDegree & $\%$ OUT & $\%$ IN \\
\hline Andalucía & 7.804 & 11,40 & 34 & 98 & 3,85 & 11,10 \\
\hline Aragón & 2.880 & 4,20 & 54 & 54 & 6,12 & 6,12 \\
\hline Asturias & 2.558 & 3,70 & 55 & 35 & 6,23 & 3,96 \\
\hline Castilla y León & 3.868 & 5,70 & 51 & 48 & 5,78 & 5,44 \\
\hline Castilla-La Mancha & 2.098 & 3,10 & 76 & 20 & 8,61 & 2,27 \\
\hline C. Valenciana & 6.893 & 10,10 & 38 & 86 & 4,30 & 9,74 \\
\hline Cantabria & 1.901 & 2,80 & 76 & 22 & 8,61 & 2,49 \\
\hline Cataluña & 10.138 & 14,80 & 26 & 136 & 2,94 & 15,40 \\
\hline Extremadura & 1.101 & 1,60 & 50 & 14 & 5,66 & 1,59 \\
\hline Galicia & 3.847 & 5,60 & 38 & 45 & 4,30 & 5,10 \\
\hline Islas Baleares & 1.449 & 2,10 & 67 & 15 & 7,59 & 1,70 \\
\hline Islas Canarias & 2.160 & 3,20 & 43 & 22 & 4,87 & 2,49 \\
\hline La Rioja & 403 & 0,60 & 79 & 3 & 8,95 & 0,34 \\
\hline C. de Madrid & 13.631 & 20,00 & 31 & 189 & 3,51 & 21,40 \\
\hline Murcia & 2.077 & 3,00 & 50 & 24 & 5,66 & 2,72 \\
\hline Navarra & 2.020 & 3,00 & 57 & 27 & 6,46 & 3,06 \\
\hline País Vasco & 3.464 & 5,10 & 58 & 45 & 6,57 & 5,10 \\
\hline
\end{tabular}




\section{FIGURA 1}

Lazos de colaboración entre las regiones españolas ( $>=5 \%$ de sus documentos) (WoS 2001-2005)

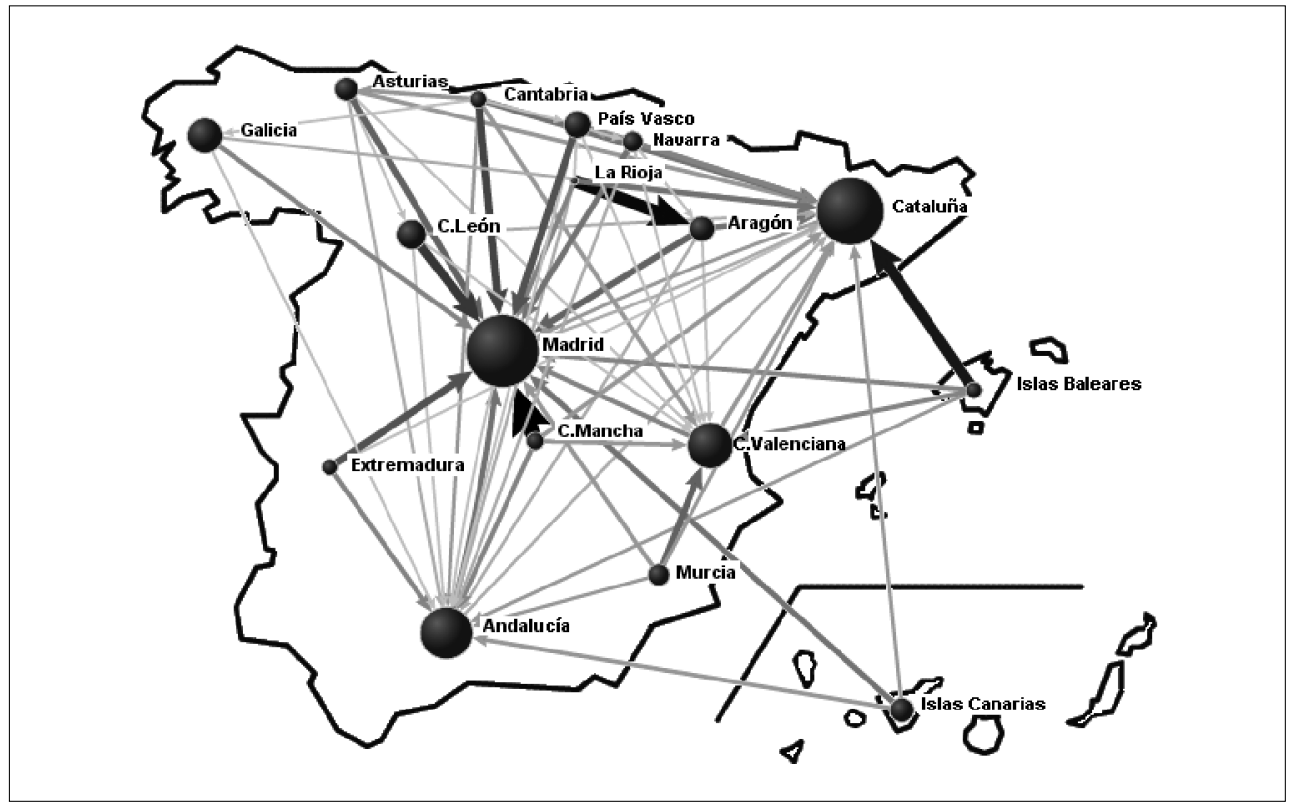

Nota: el tamaño de los nodos representa el número de documentos y el grueso y el color de las líneas representa la intensidad de las relaciones.

lla-La Mancha, con un peso mayor que el que Aragón ejerce sobre La Rioja y Cataluña sobre las Islas Baleares (Figura 1).

\section{3. ¿La colaboración con Madrid aumenta el impacto y la visibilidad por áreas y disciplinas?}

Para estudiar la posible mejora del impacto y las citas de otras regiones cuando colaboran con Madrid es necesario primero conocer la posición de Madrid respecto a España por áreas (FIR y citas relativas). De este modo, se puede valorar en qué medida Madrid ayuda a otras regiones en sus áreas fuertes (Figura 2). Madrid destaca por producción en todas las áreas excepto en Ciencias Sociales y Medicina Clínica. Por Factor de Impacto Relativo (FIR) y por Citas relativas $(C R)$ con respecto a la media de España, Madrid sobresale en todas las áreas menos en Química y se distingue en particular en Matemáticas y Biomedicina.

La colaboración con Madrid mejora generalmente el impacto (FIR) de otras regiones, excepto en Matemáticas y en Física. El caso de Física es normal, pues 
FIGURA 2

Factor de Impacto y Citas relativas de Madrid frente a España por áreas (WoS 2001-2005)

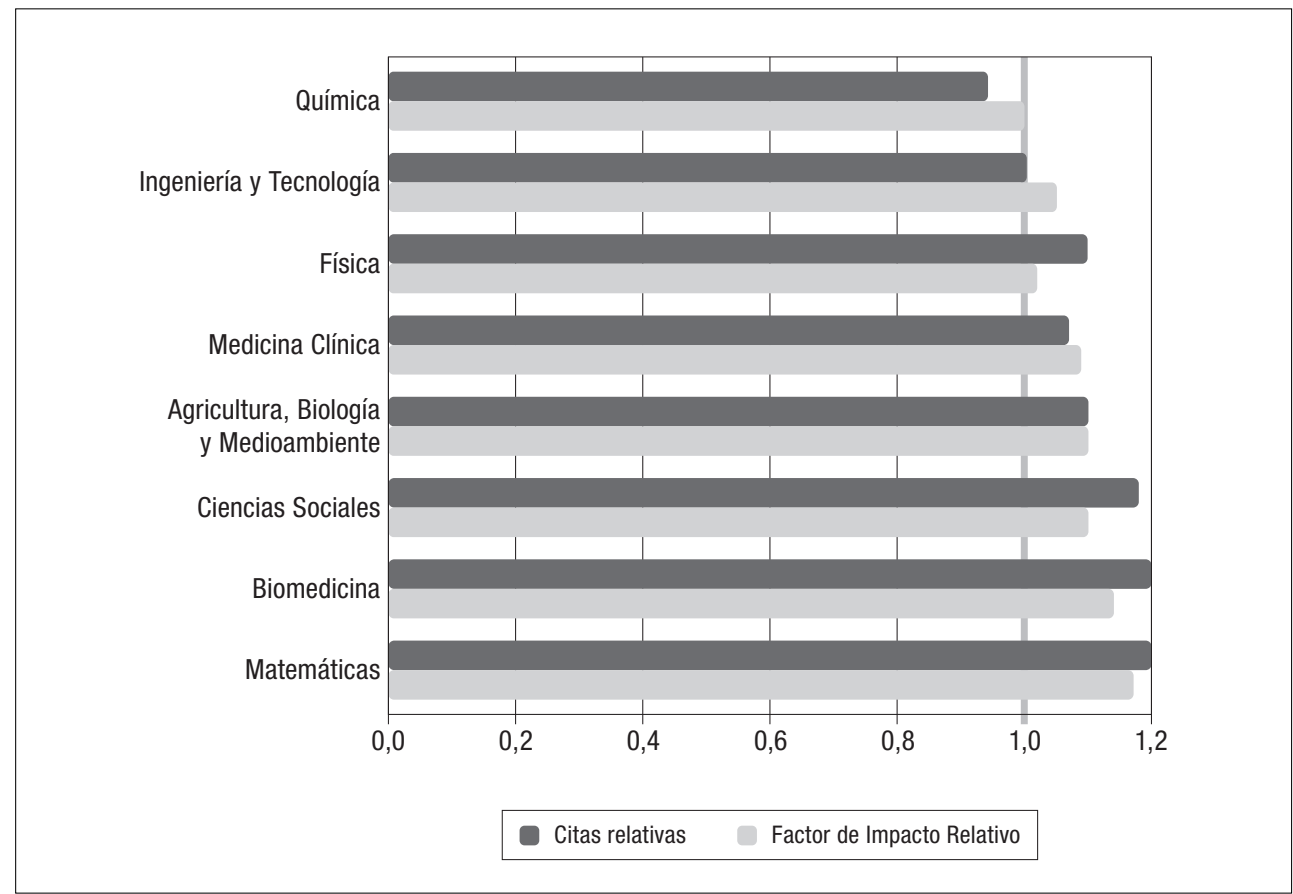

la cooperación internacional es probablemente más beneficiosa para esta área debido a sus hábitos de publicación. Además, la colaboración con Madrid mejora las citas $(C R)$, excepto en Química o Ingeniería (Tabla III). Si se considera la colaboración de Madrid con cada una de las dieciséis regiones españolas y cada una de las ocho áreas, mejora el impacto en el 75\% y la citación en el 62\% de los casos, mejorando ambos en el $51 \%$ de las ocasiones.

En la colaboración con cada región, los casos más excepcionales suceden en Medicina Clínica y en Ciencias Sociales, aunque Madrid no sea la región principal en estas áreas. También destacan Agricultura y Biomedicina: once y diez regiones respectivamente se ven favorecidas al colaborar con Madrid tanto en Factor de Impacto como en citas relativas. En el caso de Biomedicina, además, todas tienen más del 9\% de sus documentos en colaboración y, siete de estas regiones, más del 11\%.

En cuanto a las regiones, Andalucía y Cataluña se ven en general beneficiadas por la colaboración con Madrid y, más significativamente, la Comunidad Valenciana por sus elevados incrementos en impacto y citas (Tabla III). La Rioja es un caso único porque sus puntuaciones mejoran en el 75\% de los casos y, en más 


\section{TABLA III}

Factor de Impacto (FIR) y Citas relativas (CR) de la colaboración de cada comunidad con Madrid versus la producción total de cada comunidad por áreas temáticas (celdas sombreadas con FIR o CR > 1) (WoS 2001-2005)

\begin{tabular}{|c|c|c|c|c|c|c|c|c|c|c|c|c|c|c|c|c|}
\hline \multirow{2}{*}{$\begin{array}{l}\text { Comunidad } \\
\text { Autómoma }\end{array}$} & \multicolumn{2}{|c|}{ Agri } & \multicolumn{2}{|c|}{ Biom } & \multicolumn{2}{|c|}{ Fís } & \multicolumn{2}{|c|}{ Ing } & \multicolumn{2}{|c|}{ Matem } & \multicolumn{2}{|c|}{ MClin } & \multicolumn{2}{|c|}{ Quím } & \multicolumn{2}{|c|}{ Soc } \\
\hline & FIR & $C R$ & FIR & $C R$ & FIR & $C R$ & FIR & $C R$ & FIR & $\boldsymbol{C R}$ & FIR & $C R$ & FIR & $C R$ & FIR & $C R$ \\
\hline Andalucía & & & & & & $\mathrm{X}$ & & & & & $\mathrm{X}$ & $\mathrm{X}$ & & & $\mathrm{X}$ & \\
\hline Aragón & & & & & & $\mathrm{X}$ & $\mathrm{X}$ & $\mathrm{X}$ & & & $\mathrm{X}$ & & & & $\mathrm{X}$ & \\
\hline Asturias & & & & & & & & & & & & $\mathrm{X}$ & & & $\mathrm{X}$ & $\mathrm{X}$ \\
\hline C. y León & & & & & & & & & & & & $\mathrm{X}$ & & & $\mathrm{X}$ & $\mathrm{X}$ \\
\hline C.-La Mancha & & & & & & & & & & & & $\mathrm{X}$ & & & $\mathrm{X}$ & \\
\hline C. Valenciana & & $\mathrm{X}$ & & & & $\mathrm{X}$ & $\mathrm{X}$ & $\mathrm{X}$ & & $\mathrm{X}$ & & & & & $\mathrm{X}$ & \\
\hline Cantabria & $\mathrm{X}$ & $\mathrm{X}$ & & & & $\mathrm{X}$ & & & & & $\mathrm{X}$ & & $\mathrm{X}$ & & & \\
\hline Cataluña & & & & & & & & & & & & & & & $\mathrm{X}$ & $\mathrm{X}$ \\
\hline Extremadura & & & & & & & & & & & $\mathrm{X}$ & & & & $\mathrm{X}$ & $\mathrm{X}$ \\
\hline Galicia & & & & & & & & & & & $\mathrm{X}$ & $\mathrm{X}$ & & & $\mathrm{X}$ & \\
\hline Islas Baleares & & & & & & & & & & & $\mathrm{X}$ & & $\mathrm{X}$ & & & \\
\hline Islas Canarias & & $\mathrm{X}$ & & & & & & & & & & $\mathrm{X}$ & & & & \\
\hline La Rioja & $\mathrm{X}$ & $\mathrm{X}$ & & $\mathrm{X}$ & $\mathrm{X}$ & & $\mathrm{X}$ & $\mathrm{X}$ & $\mathrm{X}$ & & & $\mathrm{X}$ & & & $\mathrm{X}$ & \\
\hline Murcia & & & & & & & & & $\mathrm{X}$ & $\mathrm{X}$ & $\mathrm{X}$ & & & & & $\mathrm{X}$ \\
\hline Navarra & & $\mathrm{X}$ & & & & & & & $\mathrm{X}$ & $\mathrm{X}$ & & & & & $\mathrm{X}$ & $\mathrm{X}$ \\
\hline País Vasco & & & & & & & & & & & & & & & $\mathrm{X}$ & $\mathrm{X}$ \\
\hline
\end{tabular}

Nota: celdas marcadas con X con FIR o $C R>1,3$.

del 50\%, con valores por encima de 1,3. En particular, destaca el elevado impacto relativo en Ingeniería $(2,47)$.

Castilla-La Mancha destaca en la colaboración con Madrid en Medicina Clínica principalmente, porque presenta más del 37\% de sus documentos en colaboración con esta región, al igual que en Ciencias Sociales con un significativo aumento de las citas en el primer caso $(1,59)$ y del impacto en el segundo $(1,33)$. Otros incrementos significativos se encuentran en las Ciencias Sociales de Aragón (FIR de 2,00) o en la Física de la Comunidad Valenciana (citas relativas de 2,95).

Teniendo en cuenta las disciplinas, de las 214 en las que publican las regiones españolas, Madrid destaca por producción en 103 disciplinas, sobre todo de las áreas de Ingeniería y Matemáticas, con una actividad superior a la media de España en 86 disciplinas. Por Factor de Impacto y citas relativas, sobresale en las 
áreas de Matemáticas y Biomedicina. Por Factor de Impacto Relativo predomina en especial en Ingeniería Multidisciplinar (también por producción y citas relativas). Por citas relativas, destaca principalmente en Psicología Clínica (también por Factor de Impacto). Y, por producción, impacto y citas, sobresale en Bioquímica Biología Molecular. En 37 disciplinas, tiene valores excepcionales de actividad, impacto y citas. La mayor parte de estas disciplinas pertenecen a las áreas de Biomedicina y de Medicina Clínica.

La colaboración de otras regiones con Madrid se produce en 203 disciplinas diferentes. Andalucía y Cataluña son las regiones con mayor número de disciplinas favorecidas por la colaboración con Madrid. En la Tabla IV se muestra el número de disciplinas que mejora su FIR o $C R$ en cada región cuando colabora

\section{TABLA IV}

Factor de Impacto (FIR) y Citas relativas (CR) de la colaboración de cada comunidad con Madrid versus la producción total de cada comunidad por disciplinas (número de disciplinas con FIR o CR > 1) (WoS 2001-2005)

\begin{tabular}{|c|c|c|c|c|c|c|c|c|c|c|c|c|c|c|c|c|}
\hline \multirow{2}{*}{$\begin{array}{l}\text { Comunidad } \\
\text { Autómoma }\end{array}$} & \multicolumn{2}{|c|}{ Agri } & \multicolumn{2}{|c|}{ Biom } & \multicolumn{2}{|c|}{ Fís } & \multicolumn{2}{|c|}{ Ing } & \multicolumn{2}{|c|}{ Matem } & \multicolumn{2}{|c|}{ MClin } & \multicolumn{2}{|c|}{ Quím } & \multicolumn{2}{|c|}{ Soc } \\
\hline & FIR & $C R$ & FIR & $C R$ & FIR & $C R$ & FIR & $C R$ & FIR & $C R$ & FIR & $C R$ & FIR & $C R$ & FIR & $C R$ \\
\hline Andalucía & 10 & 6 & 12 & 10 & 13 & 9 & 15 & 11 & 4 & 4 & 26 & 24 & 6 & 2 & 16 & 17 \\
\hline Aragón & 10 & 5 & 8 & 6 & 12 & 7 & 15 & 8 & 2 & 2 & 20 & 18 & 3 & 5 & 9 & 2 \\
\hline Asturias & 13 & 7 & 13 & 8 & 8 & 7 & 12 & 7 & 1 & 0 & 21 & 18 & 1 & 4 & 8 & 6 \\
\hline C. y León & 8 & 10 & 10 & 15 & 5 & 5 & 20 & 12 & 2 & 2 & 26 & 23 & 3 & 5 & 8 & 8 \\
\hline C.-La Mancha & 11 & 12 & 13 & 9 & 8 & 7 & 15 & 9 & 1 & 2 & 19 & 24 & 3 & 4 & 4 & 3 \\
\hline C. Valenciana & 9 & 9 & 17 & 10 & 8 & 7 & 18 & 13 & 4 & 3 & 23 & 19 & 6 & 0 & 8 & 6 \\
\hline Cantabria & 4 & 3 & 7 & 6 & 4 & 4 & 11 & 5 & 2 & 2 & 16 & 14 & 2 & 1 & 4 & 2 \\
\hline Cataluña & 11 & 13 & 16 & 12 & 5 & 7 & 17 & 19 & 4 & 2 & 24 & 22 & 4 & 4 & 13 & 10 \\
\hline Extremadura & 7 & 8 & 5 & 6 & 5 & 5 & 6 & 5 & 1 & 2 & 10 & 13 & 2 & 1 & 4 & 1 \\
\hline Galicia & 15 & 13 & 12 & 10 & 12 & 7 & 14 & 11 & 2 & 2 & 24 & 17 & 6 & 4 & 12 & 3 \\
\hline Islas Baleares & 3 & 2 & 5 & 1 & 6 & 1 & 2 & 2 & 0 & 0 & 15 & 12 & 1 & 0 & 3 & 0 \\
\hline Islas Canarias & 5 & 9 & 12 & 10 & 8 & 3 & 6 & 3 & 2 & 3 & 19 & 17 & 5 & 2 & 6 & 6 \\
\hline La Rioja & 6 & 4 & 4 & 3 & 0 & 0 & 1 & 1 & 2 & 2 & 4 & 3 & 1 & 0 & 1 & 1 \\
\hline Murcia & 12 & 9 & 12 & 9 & 6 & 5 & 10 & 11 & 3 & 2 & 21 & 12 & 1 & 1 & 4 & 4 \\
\hline Navarra & 9 & 7 & 8 & 8 & 1 & 3 & 4 & 5 & 1 & 2 & 13 & 12 & 4 & 4 & 5 & 5 \\
\hline País Vasco & 8 & 3 & 11 & 8 & 10 & 8 & 20 & 12 & 2 & 3 & 18 & 16 & 5 & 2 & 7 & 8 \\
\hline Total discip. & 2 & & & 1 & & 9 & 4 & 2 & 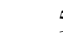 & & & & 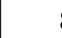 & 3 & & \\
\hline
\end{tabular}

Nota: celdas sombreadas en regiones mejoradas en más de un tercio de sus disciplinas por área. El número en cada celda indica el número de disciplinas con FIR o $C R>1$. 
con Madrid. Los datos muestran que Biomedicina y Medicina Clínica son, lógicamente, las áreas que más contribuyen a la mejora del impacto y de las citas de las regiones en el nivel de las disciplinas. Dentro de estas áreas, las disciplinas que más destacan son la Inmunología y las Enfermedades infecciosas, respectivamente. Las regiones más beneficiadas por la cooperación madrileña por impacto, citas y elevados porcentajes de colaboración (en promedio 38\% o más del total de la producción) son Castilla-La Mancha y La Rioja.

Tal como se puede apreciar en la Tabla V, las pruebas estadísticas muestran una correlación significativa entre la colaboración con Madrid y el Factor de Impacto Relativo, siendo mayor en disciplinas con más de 250 o 400 documentos.

\section{TABLA V}

Correlación en la colaboración con Madrid por disciplinas (WoS 2001-2005)

\begin{tabular}{c|l|c|c}
\cline { 3 - 3 } \multicolumn{2}{c}{} & \% Col. Madrid & FIR \\
\hline \multirow{3}{*}{ \% Col. Madrid } & Correlación de Pearson & 1 & $0,205^{*}$ \\
& Sig. (bilateral) & & 0,003 \\
& N & 205 & 203 \\
\hline \multirow{2}{*}{ FIR } & Correlación de Pearson & $0,205^{*}$ & 1 \\
& Sig. (bilateral) & 0,003 & 203 \\
\hline
\end{tabular}

* La correlación es significativa al nivel de 0,01 (bilateral).

\section{4. ¿El mayor impacto se relaciona con una mayor colaboración internacional?}

Para responder a este interrogante se estudian las disciplinas en las que publican las regiones españolas para considerar si hay correlación entre la colaboración internacional y el impacto o las citas. Como se esperaba, se encontró una correlación positiva entre todas estas variables y también con el número de documentos. Asimismo se encontró correlación positiva con la colaboración per se (incluyendo las relaciones nacionales). Esto significa que la colaboración es positiva ya sea nacional o internacional.

Cuando Madrid coopera con otras regiones hay menos colaboración internacional que cuando lo hacen sólo entre ellas. Pero, como el Factor de Impacto o las citas relativas también correlacionan con la colaboración nacional, esto no influye en los buenos resultados de la colaboración con Madrid. Cuando Madrid está presente junto a otras regiones, la mayor colaboración tiene lugar en las áreas en las que la colaboración nacional es más importante que la internacional: Biomedicina y Medicina Clínica. Además, en este último caso, para toda España, 
esta área tiene la menor colaboración internacional y las mayores proporciones de colaboración nacional.

\section{5. ¿En qué medida el análisis de redes sociales refleja los efectos de la colaboración por áreas y disciplinas?}

Considerando toda España, cuando se analizan las áreas y las disciplinas a través de las redes, se pueden observar detalladamente algunos aspectos del mapa general y de los análisis previos. Por esto, en este trabajo se estudian las redes de las áreas y disciplinas más destacadas por impacto y citas relativas: las áreas de Biomedicina y Medicina Clínica y, las disciplinas de Enfermedades infecciosas e Inmunología.

En el área de Biomedicina, Madrid es muy importante para Castilla-La Mancha, pero también para Castilla y León, Asturias, Cantabria, País Vasco, La Rioja y Aragón. Las Islas Baleares tienen relaciones más fuertes con Cataluña pero también están conectadas con Madrid (Figura 3).

En Medicina Clínica, las relaciones son más fuertes y por ello, con el mismo umbral que en Biomedicina, aparecen nuevas regiones conectadas con Madrid: Galicia, Extremadura, Andalucía, Murcia, Comunidad Valenciana e Islas Canarias.

\section{FIGURA 3}

Relaciones regionales en Biomedicina (más del 15\% de los documentos en colaboración) (WoS 2001-2005)

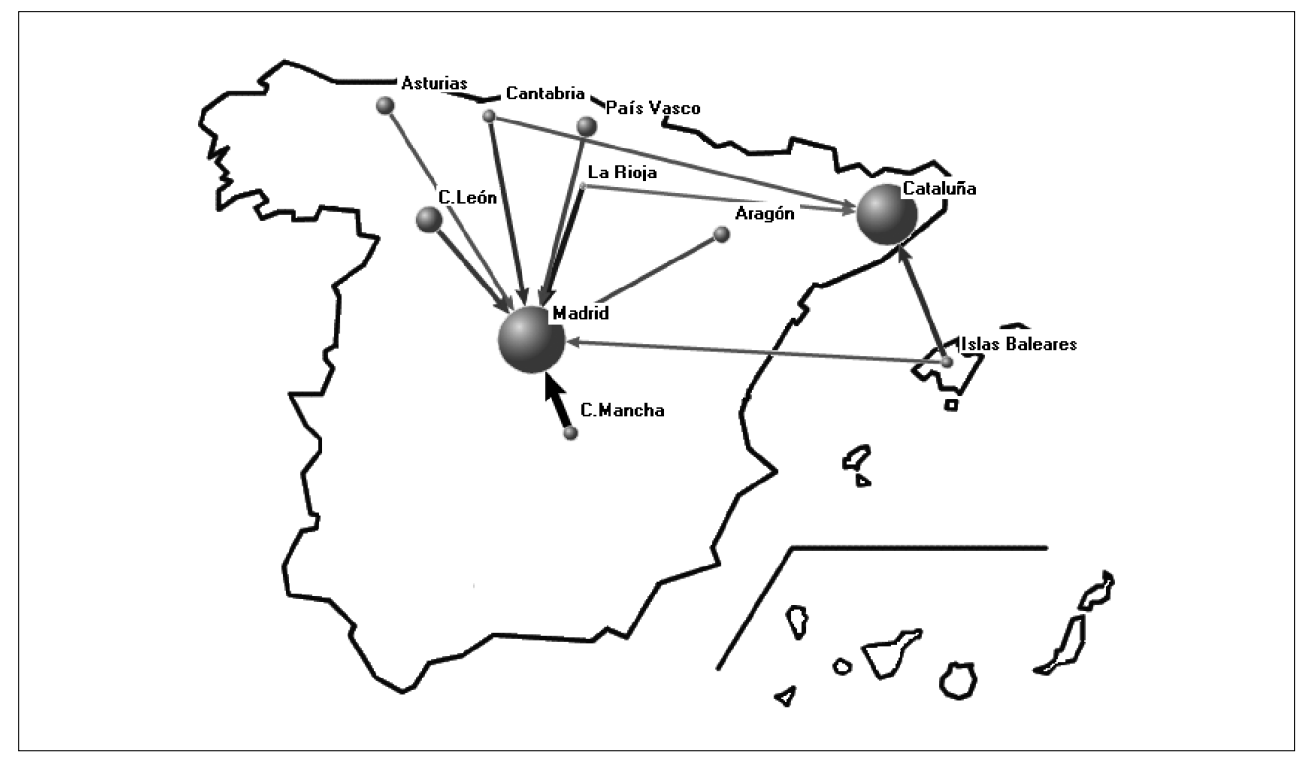

Nota: el tamaño de los nodos representa el número de documentos y el grosor y el color de las líneas representa la intensidad de las relaciones. 
Además, Cataluña es también un importante centro de atracción para otras regiones (Figura 4).

\section{FIGURA 4}

Relaciones regionales en Medicina Clínica (más del 15\% de los documentos en colaboración) (WoS 2001-2005)

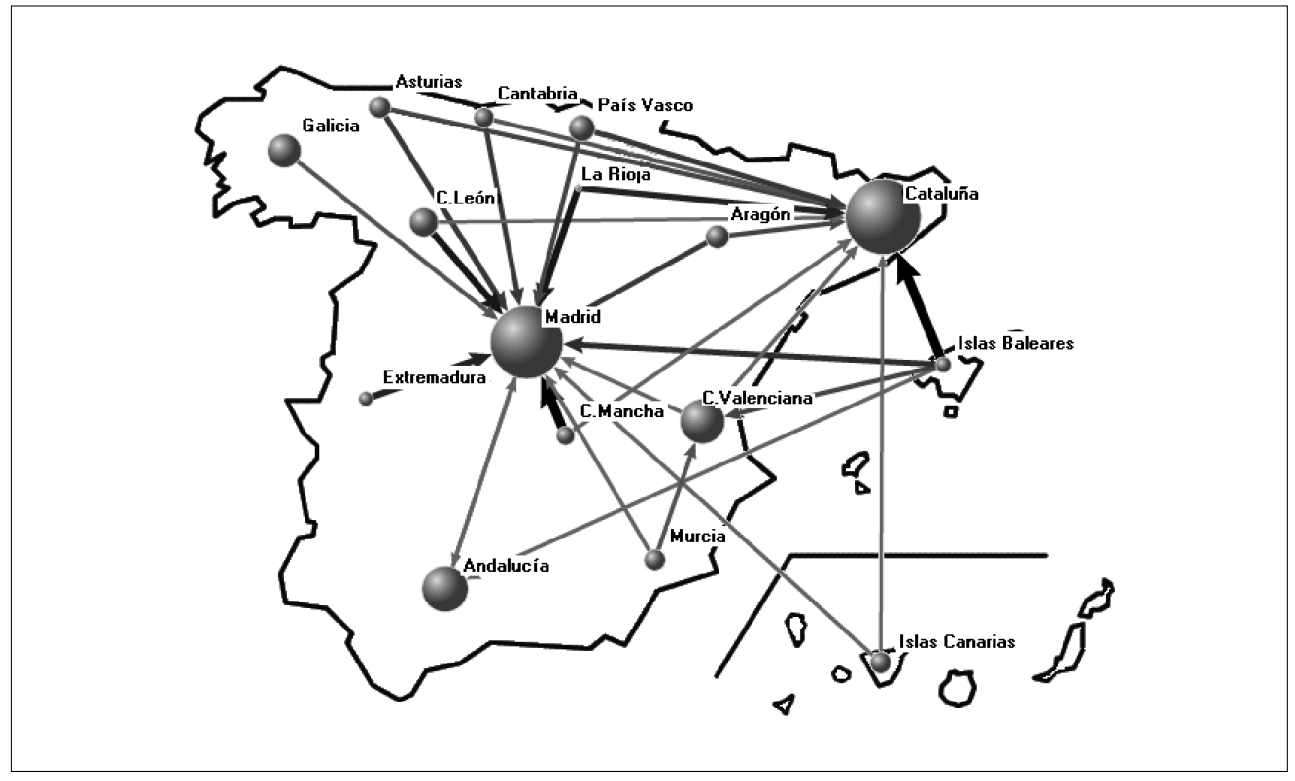

Nota: el tamaño de los nodos representa el número de documentos y el grosor y el color de las líneas representa la intensidad de las relaciones.

Teniendo en cuenta todas las relaciones, tanto en Biomedicina como en Medicina Clínica la densidad de la red es del 100\%, es decir, está totalmente conectada. Por este motivo, no tiene sentido obtener las medidas de intermediación y cercanía porque no habrá diferencias entre los nodos. Por otro lado, se obtuvieron medidas de grado de centralidad y, para Biomedicina, el porcentaje de centralidad interna (InDegree) de Madrid fue el más alto (25,19\%, más elevado que para el total de las áreas) seguido del de Cataluña (16,53\%). Para Medicina Clínica, el porcentaje de InDegree de Madrid ocupa también la primera posición (19,48\%, aunque inferior al del total de las áreas) seguido de Cataluña (16,44\%, que sí supera los valores del total de las áreas). Para Biomedicina y Medicina Clínica, los porcentajes más elevados de centralidad externa (OutDegree) son para La Rioja (10,35\% y 9,96\% respectivamente; también mayores que en el total de las áreas). Para Biomedicina, el promedio de InDegree fue del 38,06\% y el de OutDegree del 8,82\%. Para Medicina Clínica, el promedio de InDegree fue 
excepcional (40,54\%) y el de OutDegree del 12,15\%. En ambos casos, con valores más altos que España en todas las áreas.

Se estudian también las colaboraciones producidas en el nivel de las disciplinas con más del 30\% de los documentos en colaboración. Así se puede apreciar que en Inmunología, Castilla y León, La Rioja, las Islas Baleares, la Comunidad Valenciana y Castilla-La Mancha dependen de Madrid, pero hay una conexión clara entre las Islas Baleares y Cataluña y entre La Rioja y Andalucía (Figura 5).

\section{FIGURA 5}

Relaciones regionales en Inmunología (más del 30\% de los documentos en colaboración) (WoS 2001-2005)

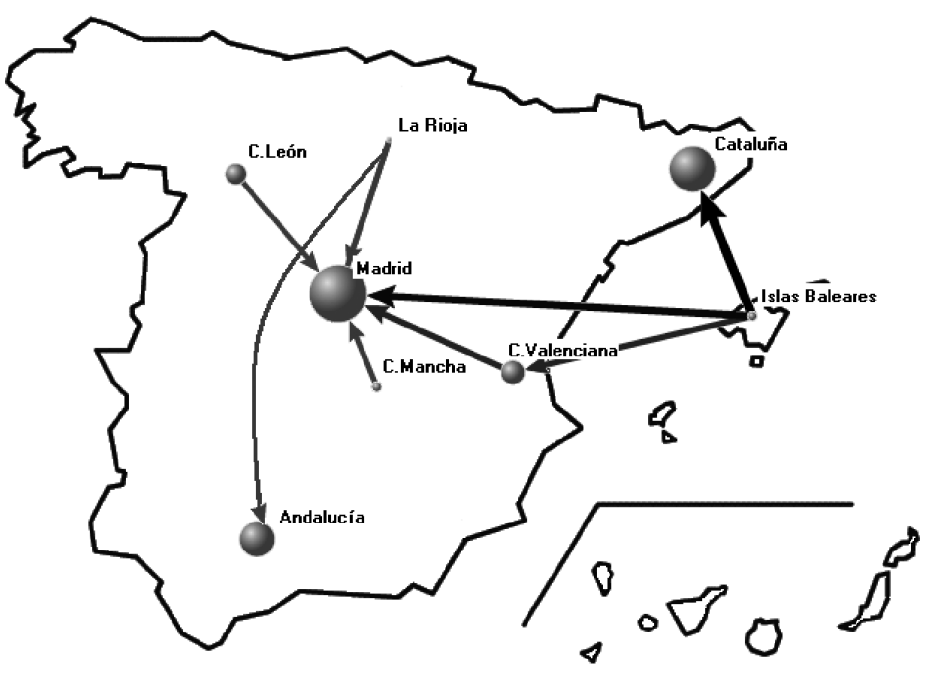

Nota: el tamaño de los nodos representa el número de documentos y el grosor y el color de las líneas representa la intensidad de las relaciones.

En Enfermedades infecciosas, Galicia, Asturias y el País Vasco aparecen en el mapa, desapareciendo a su vez Castilla y León, la Comunidad Valenciana y Andalucía (Figura 6).

Considerando todas las conexiones, en Inmunología, la red está de nuevo totalmente relacionada. No es el caso de Enfermedades infecciosas, aunque en esta disciplina se detecta una elevada densidad media (94,85\%). En Inmunología, el porcentaje de centralidad interna (InDegree) de Madrid es el más alto (20,47\%, aunque inferior al total de las áreas) y en Enfermedades infecciosas es del 19,95\%. 
FIGURA 6

Relaciones regionales en Enfermedades infecciosas (más del 30\% de los documentos en colaboración) (WoS 2001-2005)

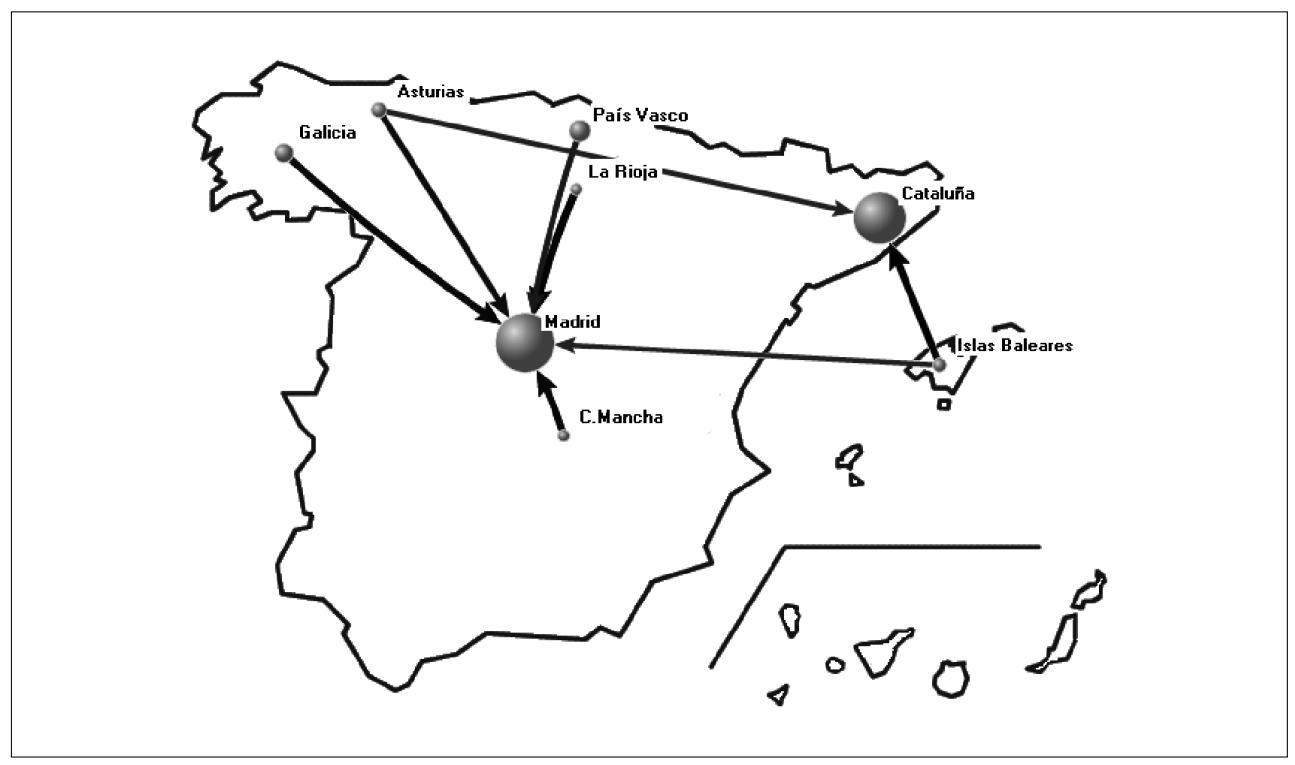

Nota: el tamaño de los nodos representa el número de documentos y el grosor y el color de las líneas representa la intensidad de las relaciones.

En ambos casos, Cataluña es la segunda región (14,46\% para Inmunología y $15,86 \%$ para Enfermedades infecciosas). Los mayores porcentajes de centralidad externa (OutDegree) son para las Islas Baleares (12,02\%), en Inmunología y, para Asturias (9,15\%), en Enfermedades infecciosas. La Rioja sigue a estas regiones con un 8,92\% en Inmunología y un 8,78\% en Enfermedades infecciosas. El promedio de InDegree en Enfermedades infecciosas es notablemente más alto que en Inmunología (48,29\% versus 36,81\%). Sin embargo, el promedio de OutDegree es mayor en Inmunología (15,48\%) que en Enfermedades infecciosas $(11,23 \%)$.

Si se estudian los centros que publican en la disciplina de Enfermedades infecciosas (Figura 7), en Madrid, los hospitales de La Paz y de Gregorio Marañón son clave para los hospitales de Virgen de la Luz de Cuenca y de Infanta Elena de Huelva. Estos dos también están conectados con los hospitales de Santa Cruz y San Pablo y el de Vall D'Hebrón de Barcelona.

La densidad media de la red es del 5,74\% y el promedio del grado de intermediación es del 13,29\%. Con un grado de intermediación normalizada más alta destacan: el Centro Nacional de Microbiología de Madrid (CNM), la Ciutat Sanitaria Vall D’Hebrón de Barcelona y el Instituto de Salud Carlos III de Ma- 
drid. El Vall D’Hebrón sobresale otra vez con el más alto grado de cercanía normalizado. Se obtienen las medidas del grado de centralidad y los porcentajes más altos de centralidad interna (InDegree) son para los que destacaban por grado de intermediación normalizado. Sin embargo, la atracción proporcional está bastante repartida: el promedio de InDegree es del 6,24\%, menos que el del centralidad externa (OutDegree del 9,92\%). La ausencia del CNM en el mapa (Figura 7) se explica por el alto umbral de relaciones exigido. Solamente se dibujan las colaboraciones más fuertes para apreciar mejor las conexiones más excepcionales. Este centro tiene algunas relaciones fuertes con centros pequeños (menos de 5 documentos) o muchas relaciones débiles con centros grandes.

\section{FIGURA 7}

Relaciones entre centros en Enfermedades infecciosas (50\% o más de los documentos en colaboración) (WoS 2001-2005)

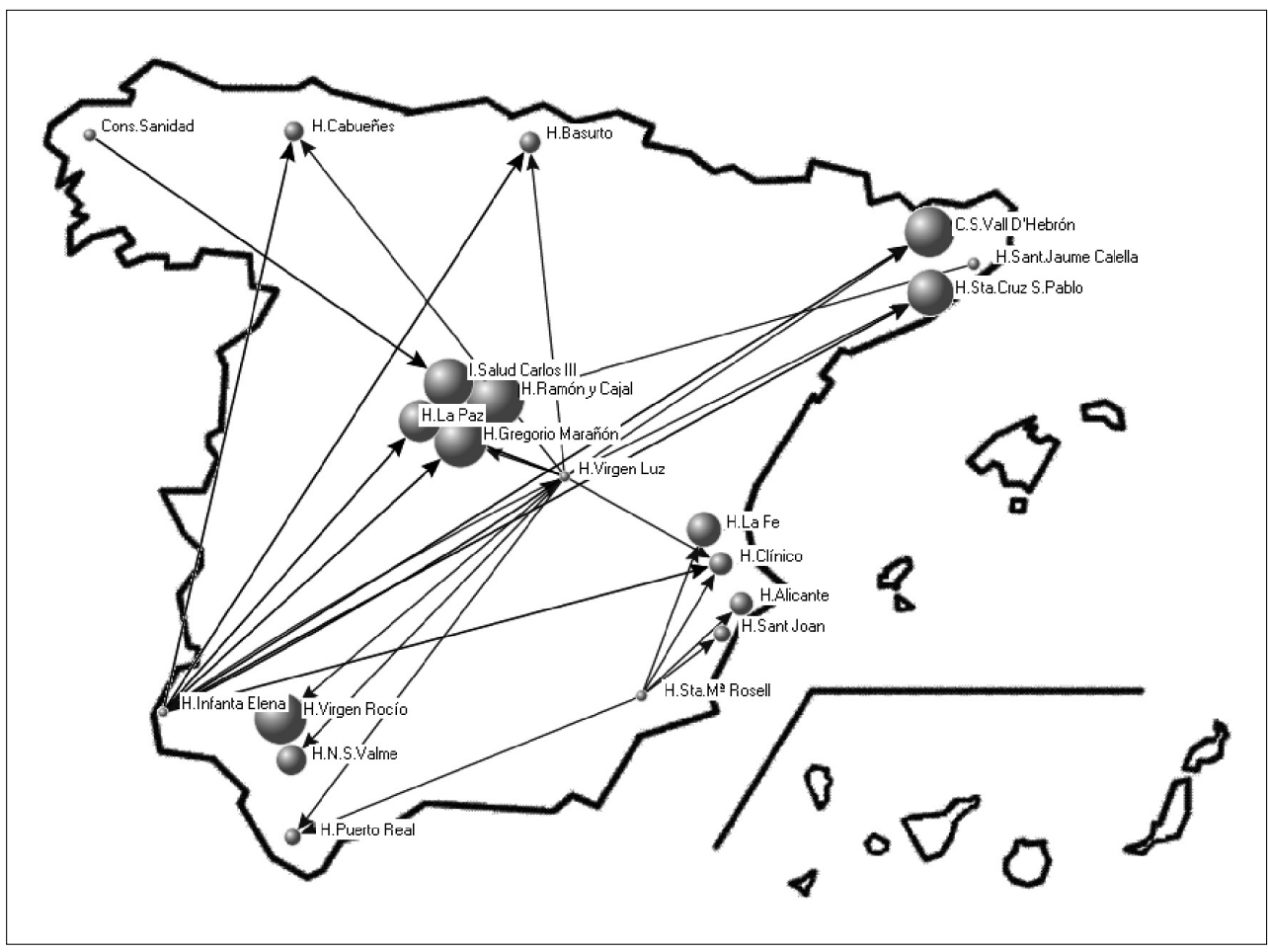

Nota: nodos de cinco o más documentos. El tamaño de los nodos representa el número de documentos y el grosor y el color de las líneas representa la intensidad de las relaciones. 


\section{Discusión y conclusiones}

Al analizar la distribución regional de la producción científica en distintos países, se observan patrones similares. En el caso de Francia, por ejemplo, como comentan Okubo y Zitt (2004), la región de París (Ile-de-France) tiene considerable peso científico y muestra un aspecto particularmente centralizado de la estructura de ciencia y tecnología francesas. Esta región no tiene ninguna frontera internacional, pero no está lejos de la frontera norte de Francia. Ile-de-France produce el 38\% de las publicaciones científicas francesas mientras que las diez regiones fronterizas originan otro $45 \%$ y las regiones interiores solamente el 17\%. Markusova y otros (2004) observan también ese particular patrón de colaboración en las regiones rusas situadas en las fronteras. En su trabajo perciben relaciones específicas entre las universidades del extremo oriente ruso y las universidades japonesas y chinas.

Como en Francia, dos Santos y Rumjanek (2001) presentan una imagen centralizada para Brasil. Alrededor del $80 \%$ de los artículos de Inmunología proceden de cuatro estados (São Paulo, Río de Janeiro, Minas Gerais y Bahía), siendo São Paulo responsable de más de la mitad de esos artículos. Este predominio de São Paulo se ha observado en diversas áreas y puede ser reflejo de la financiación de la investigación así como de la tradición investigadora de sus instituciones.

De la misma manera, España ha emergido como un importante referente científico en Europa en las dos últimas décadas, mientras que su actividad en ciencia y tecnología se concentra particularmente en las regiones de Madrid y de Barcelona que representan más de la mitad de la producción española (Gómez y otros, 2007).

Aunque el volumen de artículos coautorados con investigadores internacionales se ha duplicado en una década, Okubo y Zitt (2004) encuentran razones para pensar que el efecto de la internacionalización en la colaboración no debe ser sobrestimado. La estructura de la red de colaboración se asienta sobre una base cultural y política, y las redes de mutua afinidad siguen siendo asombrosamente estables, por lo menos para los grandes países europeos, a pesar del enérgico desarrollo de flujos de colaboración en lo que a volumen se refiere. La producción coautorada sigue siendo en gran parte un tema nacional, y la generación de artículos en colaboración internacional se presenta como un medio inusual de creación del conocimiento, aunque esto varía mucho de unas áreas a otras. En el caso de Francia, el fuerte peso de la región de París tanto en publicación como en coautoría internacional también explica este resultado, como sucede en España con las regiones de Madrid y Barcelona. Tal como demuestran Okubo y Zitt, la colaboración se da en primer lugar con vecinos nacionales, luego con otros nacionales y con regiones vecinas extranjeras y, finalmente, con otras regiones en países vecinos o con regiones en países no vecinos.

En este trabajo, se ha estudiado la influencia de Madrid en otras regiones a partir de la constatación de la tendencia hacia la descentralización ocurrida en los últimos 25 años. En este contexto, se aprecia que Madrid está perdiendo gra- 
dualmente su papel central para dar paso a otras regiones, debido principalmente a las nuevas universidades que actúan como polos de atracción científica (Gómez y otros, 2005). Sin embargo, Madrid juega un rol importante en la actividad científica española. Esto se puede comprobar no sólo mediante el recuento completo de los documentos sino también mediante el recuento fraccionado porque no hay diferencias significativas entre ambos métodos. Las regiones que más aumentaron su producción durante el período 1996-2005 fueron la Comunidad Valenciana y Andalucía pero, proporcionalmente, el crecimiento más excepcional lo experimentó Castilla-La Mancha. La colaboración con Madrid es muy significativa para las regiones menos desarrolladas, aunque no sea responsable directa del crecimiento de las mismas. Y Cataluña ejerce, también, de importante centro de atracción. La interacción entre las pequeñas y grandes regiones también obedece a la necesidad de las pequeñas de acceder a recursos especializados económicos, humanos y materiales. Gómez y otros (2005) señalan que el nuevo conocimiento puede ser la base de la innovación, que lleva a aumentar el desarrollo económico y el desarrollo regional. Esta dinámica se nota no sólo en la colaboración interna de los países sino también en las interacciones externas, como muestran Russell y otros (2007) en su estudio de la colaboración regional en América Latina.

En el artículo de Meneghini (1996), la producción científica brasileña y su impacto internacional aumentaron considerablemente en los últimos 10 años a pesar de la reducción de los recursos científicos durante el mismo período. Esta aparente paradoja se sustenta sobre el activo proceso de colaboración internacional y nacional. También es revelador que la colaboración local tenga un efecto significativo sobre las tasas de citación, aunque en menor medida que la colaboración internacional. A través del estudio de las citas, De Filippo y otros (2008) detectan también una relación entre el impacto y el número de países firmantes, lo que pone de manifiesto la importancia de la colaboración. En el caso de la colaboración de Madrid con otras regiones, aunque es fundamentalmente de tipo nacional, tiene importantes valores de impacto y citas. Las pequeñas regiones establecen (en términos de Kim, 2006) una relación asimétrica que les permite complementar capacidades y obtener mayores beneficios. En general mejora el impacto de todas las áreas, excepto Matemáticas y Física. Medicina Clínica es el área más destacada (con un aporte clave de la colaboración nacional) y la Comunidad Valenciana es la región que más se beneficia al colaborar con Madrid. En el caso de las disciplinas, las regiones con mayores incrementos de impacto o citas son Andalucía y Cataluña y las disciplinas más sobresalientes las correspondientes a las áreas de Biomedicina y Medicina Clínica.

Larivière y otros (2006) argumentan que hay una dicotomía básica entre las Ciencias Naturales y la Ingeniería por una parte y, las Sociales y Humanidades por otra parte. Demuestran que, contrariamente a la creencia frecuente, los investigadores en Ciencias Sociales y Humanidades no forman una categoría homogénea. De hecho, las actividades de colaboración de los investigadores en 
Ciencias Sociales son más equiparables a las de los investigadores de Ciencias Naturales que a los de Humanidades. Además, estos autores ven que la lengua y la proximidad geográfica influyen en la elección de colaboradores en ambos casos. Dicen que dentro de Canadá la forma más común de publicación conjunta es la colaboración internacional, seguida de la colaboración inter-institucional. Esto es contrario a la apreciación de Okubo y Zitt (2004) y a nuestro caso particular de España, aunque la colaboración internacional y la nacional están bastante equilibradas (y hay una colaboración nacional e internacional mixta cada vez mayor).

Según Wagner (2005), las redes exhiben diversas dinámicas basadas en la consiguiente investigación científica. Puede haber motivaciones específicas del tema para los patrones de colaboración que no se pueden considerar mirando los datos a un nivel de agregación superior. De Filippo y otros (2008) sostienen que el análisis de redes sociales en cada área permite observar los diferentes vínculos institucionales y la diferente estructura de cada una de ellas. En este estudio se representa el gráfico de regiones para Medicina Clínica y Biomedicina. Son gráficos bastante diferentes como sugiere Wagner (2005), porque la Medicina Clínica está más fuertemente inter-relacionada. Sin embargo, también hay puntos comunes. En ambos casos, las regiones más beneficiadas por la colaboración con Madrid son Castilla-La Mancha (también por impacto o citas) y La Rioja. En Medicina Clínica, Cataluña es más claramente el centro de atracción, al igual que en el estudio de citas.

Para las disciplinas, se eligieron Enfermedades infecciosas e Inmunología y se mostraron, además, los centros más destacados de Enfermedades infecciosas. Enfermedades infecciosas, como disciplina de Medicina Clínica, es más bipolar que Inmunología y la influencia de Cataluña es mayor con un peso importante, además, de su centro más destacado: el Hospital Vall D’Hebrón. Wagner (2005) sugiere que también los profesionales buscan oportunidades de colaboración para aumentar su visibilidad dentro de su campo. En general puede decirse que las redes reflejan detalladamente lo visto en el estudio global y que pueden representar claramente las complejas relaciones entre las regiones.

En conclusión, ya sea por producción, impacto o visibilidad, las regiones centrales como Madrid, ejercen una influencia efectiva en la actividad científica de las regiones periféricas. Otros estudios de caso en otras regiones o ciudades centrales permitirán considerar en qué medida son éstas importantes para sus respectivos países. Esas regiones o ciudades pueden servir como motor para la investigación que ocurre en otras regiones o ciudades periféricas, lo que influye en el resultado final o en el progreso científico que se produce en todo el país. La colaboración internacional es importante para el avance de la ciencia pero también la colaboración nacional, porque es la base sobre la que se construyen las otras relaciones. Además, como recuerda Meneghini (1996), una excesiva fijación en la corriente principal de la ciencia internacional dejará poco espacio para la investigación de problemas más relacionados con contingencias locales que atraen menos a los científicos extranjeros. 


\section{Agradecimientos}

Este trabajo ha sido posible gracias al proyecto de PIPCYT de la Comunidad de Madrid y a algunos de sus resultados (Gómez y otros, 2007).

\section{Bibliografia}

Batagelj, V., y Mrvar, A. (2002): Pajek - Program for Large Network Analysis. http://vlado. fmf.uni-li.si/pub/networks/pajek/ [30-09-2008].

Beaver, D. De B. (2001): Reflections on scientific collaboration (and its study): past, present and future. Scientometrics, vol. 52 (3), 365-377.

Borgatti, S. P.; Everett, M. G., y Freeman, L. C. (2002): Ucinet 6 for Windows. Software for Social Network Analysis. Harvard: Analytic Technologies.

De Filippo, D.; Morillo, F., y Fernández, M. T. (2008): Indicadores de colaboración científica del CSIC con Latinoamérica en bases de datos internacionales. Revista Española de Documentación Científica, vol. 31 (1), 66-84.

Dos Santos, N. F., y Rumjanek, V. M. (2001) Brazilian immunology: One hundred years later. Scientometrics, vol. 50 (3), 405-418.

Glänzel, W. (2001): National characteristics in international scientific co-authorship relations. Scientometrics, vol. 51 (1), 69-115.

Godin, B., e Ippersiel, M. P. (1996): Scientific collaboration at the regional level: The case of a small country. Scientometrics, vol. 36 (1), 59-68.

Gómez, I; Bordons, M; Morillo, F; De Filippo, D., y Aparicio, J. (2009): Science E Technology Indicators for EULARINET. IEDCYT, Madrid.

Gómez, I.; Bordons, M.; Morillo, F., y Fernández, M. T. (2005): Regionalisation of science and technology data in Spain. Research Evaluation, vol. 14 (2), 137-148.

Gómez, I.; Fernández, M. T.; Bordons, M.; Morillo, F.; Aparicio, J.; Candelario, A.; De Filippo, D.; González-Albo, B.; Herrero, M., y Moreno, L. (2007): Indicadores de Producción Científica y Tecnológica de la Comunidad de Madrid (PIPCYT). Dirección General de Universidades e Investigación. Consejería de Educación. Comunidad de Madrid. Madrid, p. 232.

Kim, K. W. (2006): Measuring international research collaboration of peripheral countries: taking the context into consideration. Scientometrics, vol. 66 (2), 231-240

Larivière, V.; Gingras, Y., y Archambault, E. (2006): Canadian collaboration networks: A comparative analysis of the natural sciences, social sciences and the humanities. Scientometrics, vol. 68 (3), 519-533.

Leydesdorff, L., y Wagner, C. (2006): Is the United States losing ground in science? A global perspective on the world science system. http://users.fmg.uva.nl/lleydesdorff/US Science/US Science.pdf [15-10-2008].

Markusova, V. A; Minin, V. A.; Libkind, A. N.; Jansz, C. N. M.; Zitt, M., y Bassecoulard-Zitt, E. (2004): Research in non-metropolitan universities as a new stage of science development in Russia. Scientometrics, vol. 60 (3), 365-383.

Meneghini, R. (1996): The key role of collaborative work in the growth of Brazilian science in the last ten years. Scientometrics, vol. 35 (3), 367-373. 
Moed, H. F. (2000): Bibliometric indicators reflect publication and management strategies. Scientometrics, vol. 47 (2), 323-346.

Okubo, Y., y Zitt, M. (2004): Searching for research integration across Europe: a closer look at international and inter-regional collaboration in France. Science and Public Policy, vol. 31 (3), 213-226.

Otte, E., y Rousseau, R. (2002): Social network analysis: a powerful strategy, also for the information sciences. Journal of Information Science, vol. 28 (6), 441-453.

Russell, J. M.; Ainsworth, S.; Del Río, J. A.; Narváez-Berthelemot, N., y Cortés, H. D. (2007): Colaboración científica entre países de la región latinoamericana. Revista Española de Documentación Científica, vol. 30 (2), 180-198.

Schubert, A.; Glänzel, W. (2006): Cross-national preference in co-authorship, references and citations. Scientometrics, vol. 69 (2), 409-428.

Van Raan, A. F. J. (1998): The influence of international collaboration on the impact of research results. Scientometrics, vol. 42 (3), 423-428.

Wagner, C. (2005): Six case studies of international collaboration in science. Scientometrics, vol. $62(1), 3-26$. 